\title{
Reconfiguration Strategies for Hybrid Systems
}

\author{
Kazuro Tsuda ${ }^{1}$, Domenico Mignone, Giancarlo Ferrari-Trecate, Manfred Morari \\ Institut für Automatik, ETH - Swiss Federal Institute of Technology \\ CH 8092 Zürich, Switzerland \\ Tel: +41 1632 7626, Fax: +41 1632 1211, E-mail: \{tsuda,mignone,ferrari,morari\}@aut.ee.ethz.ch
}

\begin{abstract}
In this work we propose several algorithms to solve the reconfiguration problem for linear and hybrid systems. In particular, we consider the decision about the usage of redundant hardware in order to compensate for faults. While this problem can be translated into a constrained model predictive control framework, the computational complexity grows very fast, as the number of possible decisions increases. In this work we propose schemes, that require low computational effort. We discuss the applicability of the methods considering the reconfiguration of the three tank benchmark system.
\end{abstract}

Keywords: Hybrid Systems, Fault Tolerant Control, Reconfiguration, Model Predictive Control

\section{Introduction}

Many control systems are subject to faults or malfunctions. The failure of components, like sensors or actuators can partially or fully disable the action of the controller. This results in an unsatisfactory performance of the system, decreased availability, emergency shutdowns or even significant damages to the plant and to its environment. For this reason, several research activities recently focused on the safety aspects of control systems $[8,6]$. One way to classify the different approaches is the distinction between passive and active fault accommodation [16]. In passive fault accommodation the closed loop system is designed, such that it is robust, within some performance range, to fault occurrences. On the other hand, active fault accommodation aims at changing the control operation when a fault is detected. These changes can comprise reconfiguration of the controller scheme, modifications of controller parameters or alternative setpoint trajectories. In this work we will focus on active fault accommodation. The key issue we are considering is the usage of redundant hardware for reconfiguration when some faults occur. The term hardware redundancy is used for systems where a replication of hardware components is present [16]. In other words, such systems

\footnotetext{
${ }^{1}$ on leave from: NKK Corporation, Sensing \& Control Research Department, Applied Technology Research Center 11; Minamiwatarida-cho; Kawasaki-ku Kawasaki-shi, 210-0855 JAPAN; E-mail: tsuda@lab.keihin.nkx.co.jp
}

have components that need not to be used in order to meet nominal control specifications, but can be used in extraordinary situations. The redundancy is often given by subsystems with similar functions, different parameters or alternative locations within the system topology.

The main contribution of this work is to propose several solutions for the decision problem about the choice of redundant hardware using mathematical optimization tools. We will refer to this decision simply as reconfiguration of the control scheme. To this purpose we are using the modeling formalism of Mixed Logic Dynamical (MLD) systems introduced in $[5,4]$. The equations describing an MLD system are

$$
\begin{gathered}
x(t+1)=A x(t)+B_{1} u(t)+B_{2} \delta(t)+B_{3} z(t)+B_{6} \phi(t) \\
y(t)=C x(t)+D_{1} u(t)+D_{2} \delta(t)+D_{3} z(t)+D_{6} \phi(t) \\
E_{2} \delta(t)+E_{3} z(t) \leq E_{1} u(t)+E_{4} x(t)+E_{5}+E_{6} \phi(t)
\end{gathered}
$$

where $x \in \mathbb{R}^{n_{c}} \times\{0,1\}^{n_{\ell}}$ are the continuous and binary states, $u \in \mathbb{R}^{m_{c}} \times\{0,1\}^{m_{\ell}}$ are the inputs, $y \in \mathbb{R}^{p_{c}} \times\{0,1\}^{p_{\ell}}$ the outputs, and $\delta \in\{0,1\}^{r_{\ell}}, z \in \mathbb{R}^{r_{c}}$ represent auxiliary binary and continuous variables, respectively, $\phi \in\{0,1\}^{f_{\ell}}$ denote the occurrence of faults. The auxiliary variables are introduced when translating propositional logic into linear inequalities. For a detailed description of the features and modeling capabilities of MLD systems, we defer to [5, 3]. Constraints on state, input, and auxiliary variables are summarized in the inequality (1c). There are four main reasons for formulating active fault accommodation within the MLD framework. First, the framework allows to consider a large class of systems, including LTI systems, systems with discrete inputs, systems interacting with logic automata and many more. Second, the priorities for the usage of redundant components can be directly included within the MLD model and can therefore be systematically considered during computations. Third, these models are suitable for formulating control or estimation problems as mathematical programs for which solvers are commercially available. Fourth, the decision about redundant hardware is an inherently binary problem, therefore the modeling with binary variables is appropriate. Throughout the paper, the three tank system in Figure 1 will be used to illustrate the proposed ideas. The reconfiguration problem for the three tank system has been defined as a COSY 
benchmark problem [11, 9]. For instance in [1] and [12] schemes that only take into account qualitative information about the state of the plant are considered. In [17] the reconfiguration is formulated as a multi-model switching control task. Approaches using neural networks are reported in [13]. The paper is organized as follows: In Section 2 we summarize the model of the three tank system. In Section 3 the main issues for fault detection and control of MLD systems are presented. In Section 4 we present the ideas for reconfiguration of MLD systems. In Section 5 we apply some of the algorithms to the tank system. The complete version of this paper can be found in [18].

\section{The Three Tank System}

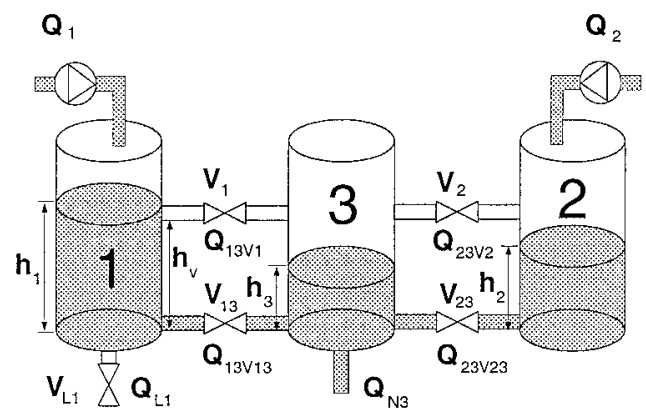

Figure 1: The COSY Three Tank Benchmark

The three tank system represented in Figure 1 has been adopted recently as a standard benchmark problem for fault detection and reconfigurable control [11]. Three tanks can be filled with two independent pumps acting on the tanks 1 and 2. The tanks are interconnected to each other with switching valves. The nominal outflow $Q_{N 3}$ from the tanks is placed at tank 3 . In closed loop operation, the controlled variables are the levels in tanks 1 and 3 . The level $h_{1}$ is controlled by a. PI controller manipulating the flow $Q_{1}$, while for the level $h_{3}$, a switching controller manipulating valve $V_{1}$ is used. In nominal operation, all other valves are closed, and the flow $Q_{2}$ is zero. Therefore the set $V_{N}$ of nominal manipulated variables for this system is given by the valve $V_{1}$ and the pump $Q_{1}$, i.e. $V_{N}=\left\{V_{1}, Q_{1}\right\}$. All other inputs form the set $V_{R}$ of redundant manipulated variables, i.e. $V_{R}=\left\{V_{13}, V_{2}, V_{23}, Q_{2}\right\}$. In $[14,4]$ it is shown how this system can be modeled in MLD form. The following fault scenarios [11] are considered:

Scenario 1: Valve $V_{1}$ is stuck open;

Scenario 2: Valve $V_{1}$ is stuck closed;

Scenario 3: Tank 1 has a leak $Q_{L 1}$

For each of these faults, the aim of the reconfiguration scheme is to keep the level $h_{1}$ at 0.5 and $h_{3}$ at 0.1 , by possibly using the redundant manipulated variables $V_{R}$. In scenario 3 the additional goal is to have a minimal water loss from tank 1 . There are some aspects that complicate the reconfiguration task for this system:

- There are several possibilities for the choice of the redundant hardware. To check and evaluate all combinations represents a large combinatorial problem.

- The choice of redundant hardware has to be made fulfilling a hierarchy of preferences.

- The plant exhibits typical characteristics of a hybrid system. The dynamics change, if the liquid reaches the height of the upper valves. Moreover, the model has to take into account discrete inputs because of the presence of switching valves.

- The control goal in nominal operation can only be reached by providing a sequence of periodic inputs to the system. For some reference values no constant steady state exists, if the nominal hardware $V_{N}$ is used.

- The system is subject to constraints on the liquid levels $h_{i}$ and on the inflows $Q_{j}$.

\section{Fault Detection and Control}

\subsection{Problem Formulation for Reconfiguration}

The supervision scheme we are considering consists of two stages: In a first stage a fault detection and isolation algorithm is required to monitor the operation of the plant. This part should estimate at each time step which faults occurred or that no fault was detected. We assume that this task is already accomplished, for instance by using the tools described in [4]. The second stage is the actual reconfiguration component. The conceptual scheme is shown in Figure 2. The reconfig-

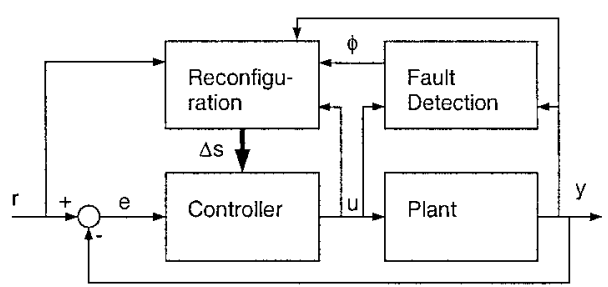

Figure 2: Reconfiguration Scheme

uration device should decide, which system modification is most suitable to achieve the control goals, or to achieve the best possible performance in terms of some value function. The reconfiguration block takes as inputs the reference $r$, the control action $u$, the output signal $y$ and the fault information $\phi$. The output is a modification of the controller structure $\Delta s$, which can be the inclusion or exclusion of some actuators or the switch to a different controller. The problems of control and fault detection for MLD systems have been considered previously in $[5,4]$. The main ideas are summarized next. 


\subsection{Predictive Control of MLD Systems}

There are several ways to systematically find a controller for MLD systems. The approach that we adopt was presented in [5] and uses the ideas of Model Predictive Control (MPC). At each time step the following constrained optimization problem is solved:

$$
\begin{gathered}
\min _{\underline{u}, \underline{\delta}, \underline{z}} J=\sum_{k=0}^{T-1}\left\|u(k+t \mid t)-u_{f}\right\|_{Q_{1}}^{2}+\left\|\delta(k+t \mid t)-\delta_{f}\right\|_{Q_{2}}^{2}+ \\
z(k+t \mid t)-z_{f}\left\|_{Q_{3}}^{2}+\right\| x(k+t \mid t)-x_{f}\left\|_{Q_{4}}^{2}+\right\| y(k+t \mid t)-y_{f} \|_{Q_{5}}^{2}
\end{gathered}
$$

subject to $(1 \mathrm{a}),(1 \mathrm{~b}),(1 \mathrm{c})$ and

$$
x(k+T \mid t)=x_{f}
$$

where $\|x\|_{Q}^{2}=x^{\prime} Q x, Q_{i}=Q_{i}^{\prime} \geq 0, i=1, \ldots, 5$, are given weight matrices, and $x_{f}, u_{f}, \delta_{f}, z_{f}, y_{f}$ are the target values of the MLD model variables. A steady state target value for an MLD system can be determined by solving the following mixed integer program [2]:

$$
\begin{aligned}
\min _{x_{f}, u_{f}, \delta_{f}, z_{f}}\left\|y_{f}-\gamma\right\|+\left.\left\|x_{f}\right\|\right|_{\rho_{4}}+\left\|u_{f}\right\|_{\rho_{1}}+\left.\left\|z_{f}\right\|\right|_{\rho_{3}}+\left\|\delta_{f}\right\|_{\rho_{2}} \\
\text { s.t. } \quad x_{f}=A x_{f}+B_{1} u_{f}+B_{2} \delta_{f}+B_{3} z_{f}+B_{6} \phi_{f} \\
E_{2} \delta_{f}+E_{3} z_{f} \leq E_{1} u_{f}+E_{4} x_{f}+E_{5}+E_{6} \phi_{f}
\end{aligned}
$$

Here $\|\|$. is an arbitrary norm, $\rho_{i}$ are small, positive definite weights. We recall one property of MLD systems: the phenomenon of cycling steady states [18]. For certain systems, the switching components and the MPC controller cause a periodic trajectory of the system around the reference. This gives lower cost in (2) than the stabilization at the constant steady state found with (4)-(6). In other words, the system goes through a cycle of states to stay close to the reference, instead of converging to a constant state. The three tank model presented in Section 2 shows this behaviour for the reference value $r=[0.5,0.0,0.1]$ the valve $V_{1}$ has to switch repeatedly in order to keep level $h_{3}$ close to its reference [18].

\subsection{Fault Detection of MLD Systems}

The fault detection problem for MLD systems has been considered in [4]. The occurrence of the $i$-th fault is denoted by $\phi_{i}=1 \mathrm{in}$ (1). The faults can be estimated by using a moving horizon estimation $[4,7]$ technique. At each time step a least squares problem extending backwards in time over a given horizon is solved. This optimization has the MLD dynamics as constraints. As in controller synthesis, the structure of this mathematical problem is a mixed integer quadratic program (MTQP) [15].

\subsection{Objective Prioritization in MLD Systems}

We require that the reconfiguration scheme is capable to respect preferences about the use of certain system components. In [10] it was shown, that such objective prioritizations can be directly specifed within the MLD framework. The key idea is to formulate the hierarchy of priorities as a set soft constraints in the MPC optimization (2),(3) yielding:

$$
\begin{array}{cc}
\min _{\theta, \epsilon, \delta} & \theta^{\prime} H \theta+f^{\prime} \theta+\epsilon^{\prime} S \epsilon+\rho M_{p}^{\prime} \delta \\
\text { s.t. } & A \theta \leq b+C \epsilon \text { and } 0 \leq \epsilon \leq M_{\epsilon}
\end{array}
$$

where $\theta=(\underline{u}, \underline{\delta}, \underline{z}), H$ and $f$ define the cost function of the MPC optimization, and all constraints are summarized in $A$ and $b . \epsilon$ is a vector of slack variables, representing the constraint violations, $C$ is a matrix of appropriate dimension, whose rows are 0 or $e_{k}^{\prime}$ depending on whether the $k^{\prime}$ th constraint is hard or soft, $S>0$ is a weighting matrix which determines the trade-off between cost and constraint violation. Additional variables $\delta \in\{0,1\}^{r}$ are defined and are associated with constraint violations at $r$ levels of priority. For details we defer to [10]. We just note that the inclusion of these priorities does not alter the optimization problem structure.

\section{Reconfiguration of MLD systems}

In this section we present various strategies for performing the decision on redundant hardware in case of faults. We characterize the manipulated variables of an MLD system as follows:

The set $V_{N}$ (Nominal Inputs) is the set of all manipulated variables used in nominal operation.

The set $V_{R}$ (Redundant Inputs) is the set of all other manipulated variables, i.e., those inputs that can be used additionally for reconfiguration.

The sets $V_{i}$ (Candidate Inputs) $\left(i=1, \ldots, n_{f}\right)$ are the sets of manipulated variables that are proposed to be used by the reconfiguration scheme when fault $\phi_{i}$ is detected. $n_{f}$ is the number of fault scenarios considered.

We assume that all manipulated variables have been partitioned into the sets $V_{N}$ and $V_{R}, V_{R}$ is nonempty, $V_{R} \cap V_{N}=\emptyset$ and $V_{i} \subset\left(V_{R} \cup V_{N}\right)\left(i=1, \ldots, n_{f}\right)$

\subsection{Reconfiguration as a Control Problem}

One way to handle the reconfiguration problem is to treat it as a control problem within a receding horizon framework. Once a fault has been detected, we carry on solving the MPC optimization (2),(3) with the corresponding fault information. In faultless mode we impose the usage of the set $V_{N}$ of manipulated variables by adding prioritizations in the control synthesis optimization, as mentioned in Section 3.4. This idea is summarized in the following reconfiguration algorithm.

\section{Algorithm 1}

1. Consider the complete model (1) of the plant, including the complete set of manipulated variables $V_{N} \cup V_{R}$.

2. While $\phi=0$, apply the MPC control algorithm in [5] to the complete model. To avoid the use of the inputs $V_{R}$ in nominal operation include prioritizations on the inputs $V_{N}$. 
3. If a fault $\phi_{i}$ occurs, update $\phi(t)$ and use it in the MPC optimization for all future time steps where the fault is active.

The advantage of this algorithm is that it gives the best possible strategy for reconfiguration, where "best" has to be understood in terms of the cost function (2) for the controller. We can prove stability [18] of this scheme as in [5, Theorem 1]. The drawback is the size of the optimization since the optimization problems for controller synthesis are done at all time instances over the complete model. Therefore the number of variables is large, a problem that is particularly pronounced in the mixed integer optimizations of MLD systems.

\subsection{Reconfiguration on Two Decision Levels}

In order to avoid the large size of the online optimization problems involved in receding horizon control, we propose three methods how to lower the computational complexity of $(2),(3)$ to the current plant operation. In this context we will measure the complexity of the models by the number of manipulated variables used in (2),(3). We assume that the receding horizon control scheme is operating with the inputs $V_{N}$, as long as no fault is detected. If a fault $\phi_{i}$ occurs, the first task of the reconfiguration procedure is to decide which inputs $V_{i}$ have to be used for control. Note that in Section 4.1 this question did not arise, since the choice of manipulated variables was implicitly given by the optimization over all available manipulated variables in $V_{N} \cup V_{R}$. The second level consists of applying the receding horizon control strategy using the manipulated variables $V_{i}$ determined in the first step. The three methods described in this section differ from each other in the first decision level, i.e., in the decision about $V_{i}$.

4.2.1 Reconfiguration with Reachability Analysis: In this strategy we propose to determine the manipulated variables $V_{i}$ offline. A look up table is set up, that denotes the set $V_{i}$ for each possible fault scenario $\phi_{i}$. The following algorithm describes the procedure:

\section{Algorithm 2}

1. Build up a look up table with the assignment of a set $V_{i}$ to each possible fault scenario $\phi_{i}$.

2. Run the fault detection scheme and the control scheme in parallel.

3. For each fault scenario $\phi_{i}$ detected, switch the model used in control according to the look up table, i.e., determine the candidate inputs $V_{i}$ from the look up table.

4. Apply the MPC algorithm using the candidate inputs $V_{i}$ as manipulated variables.

5. Repeat step 3. at the next time step using the most recent fault information.
The choice of manipulated variables $V_{i}$ can be done via reachability analysis [3]: Those inputs are chosen, that allow the target state to be reachable.

4.2.2 Reconfiguration with Static Steady State Analysis: We assume that the system does not have cycling steady states (see Section 3.2 and [18]) and that the steady state obtained by (4) to (6) is reachable. If a fault $\phi_{i}$ is detected, the candidate inputs $V_{i}$ are chosen based on the steady state optimization (4)-(6). $V_{i}$ is chosen as the set of inputs that are different from zero in this optimization. The search for the steady state can be modified to include further constraints or prioritizations, see Section 3.4. This method requires a low computational load, however it lacks guarantees of optimality of $V_{i}$. The algorithm can be summarized as follows:

\section{Algorithm 3}

1. Run the fault detection scheme and the control scheme in parallel.

2. If a fault $\phi_{i}$ occurs, run the steady-state finding procedure (4)-(6) on the model with inputs $V_{N} \cup$ $V_{R}$.

3. Choose those inputs, which are different from zero in steady state, as candidate inputs $V_{i}$.

4. Apply the MPC algorithm using the candidate inputs $V_{i}$ as manipulated variables.

5. Repeat step 2. at the next time step using the most recent fault information.

4.2.3 Reconfiguration with Polytopic Steady State Analysis: The algorithm presented here is a modification of the method shown in section 4.2.2 that can be applied to systems with cycling steady states. We assume that the cycle consists of reachable states. In case of a fault $\phi_{i}$, the method in Section 4.2.2 extracted the candidate inputs $V_{i}$ from a single steady state. For systems with cycling steady states we propose to extract $V_{i}$ from multiple steady states. For a system with $n_{y}$ outputs we solve $M=2^{n_{y}}$ optimizations, given by (4)-(6), subject to exactly one additional constraint:

$$
\mu_{k} y_{f} \leq \mu_{k} r \quad\left(k \in 1 \ldots 2^{n_{y}}\right)
$$

where

$$
\mu_{k} \in\left\{\operatorname{diag}(d) \mid d \in\left\{\left[\xi_{1}, \ldots, \xi_{n_{y}}\right] \mid \xi_{i} \in\{-1,1\}\right\}\right\}
$$

E.g. for a system with $n_{y}=2$ outputs the $M=2^{2}=4$ steady states are found by solving (4)-(6) subject to one of the following four additional constraints, respec- 
tively:

$$
\begin{array}{ll}
k=1: & {\left[\begin{array}{cc}
-1 & 0 \\
0 & -1
\end{array}\right] y_{f} \leq\left[\begin{array}{cc}
-1 & 0 \\
0 & -1
\end{array}\right] r} \\
k=2: & {\left[\begin{array}{cc}
1 & 0 \\
0 & -1
\end{array}\right] y_{f} \leq\left[\begin{array}{cc}
1 & 0 \\
0 & -1
\end{array}\right] r} \\
k=3: & {\left[\begin{array}{cc}
-1 & 0 \\
0 & 1
\end{array}\right] y_{f} \leq\left[\begin{array}{cc}
-1 & 0 \\
0 & 1
\end{array}\right] r} \\
k=4: & {\left[\begin{array}{ll}
1 & 0 \\
0 & 1
\end{array}\right] y_{f} \leq\left[\begin{array}{ll}
1 & 0 \\
0 & 1
\end{array}\right] r}
\end{array}
$$

Let $Y_{i M}$ be the set of outputs generated by the $M$ steady states computed above. We assume that

$$
r \in \operatorname{conv}\left(Y_{i M}\right)
$$

i.e., the desired reference value is element of the convex hull of the $M$ steady state outputs. If (13) is fulfilled, we define $M$ tentative sets of inputs $V_{i k} \quad(k=1 \ldots M)$ as those inputs, that are different from zero at steady state. The candidate set of inputs $V_{i}$ is then chosen such that $V_{i k} \subset V_{i} \quad(k=1 \ldots M)$. The set of inputs $V_{i}$ allows to keep the system at each of the $M$ steady state values. Therefore, if $r$ is within the polytope spanned by $Y_{i M}$, it is reasonable to use these inputs to achieve the actual goal of staying close to the reference $r$. The graphical representation of the $M=2^{2}$ steady states for the previous example is given in Figure 3.The reconfiguration algorithm is summarized next:

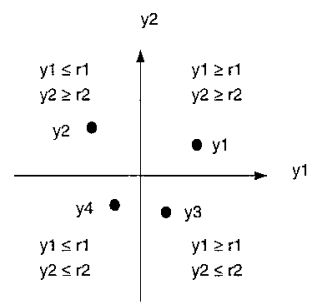

Figure 3: Outputs $Y_{i M}$ of steady states: The origin is the desired reference value $r=\left[\begin{array}{ll}r_{1} & r_{2}\end{array}\right]^{\prime}$, the outputs $y_{1}, y_{2}, y_{3}, y_{4}$ result as steady state outputs from

\section{Algorithm 4} the optimization (4)-(6), (7)

1. Run the fault detection scheme and the control scheme in parallel.

2. If the fault $\phi_{i}$ is detected, find $M$ steady states with property (13). The steady states are found with (4)-(6) on the model with inputs $V_{N} \cup V_{R}$. For each steady state define $V_{i k}$ as the set of $m a$ nipulated variables, that are nonzero in steady state.

3. Find the set of candidate inputs $V_{i}$ from $V_{i k}$.

4. Apply the MPC algorithm using the candidate inputs $V_{i}$ as manipulated variables.

5. Repeat step 2. at the next time step using the most recent fault information.

The obvious solution to find $V_{i}$ is given by $V_{i}=\cup_{k=1}^{M} V_{i k}$ However, in terms of the number of manipulated variables, this can be suboptimal. We defer to [18] for more details.

\subsection{Static Fault Compensation}

In this approach we set $V_{i}=V_{N} \cup V_{R}$ and we propose a strategy to compute the values of $V_{i}$ in case of faults, avoiding to run the optimization of Section 4.1 over all manipulated variables. We use the redundant inputs $V_{R}$ to minimize at each time step the effect of faults. For this purpose the MLD model is rewritten as:

$$
\begin{aligned}
x(t+1) & =A x(t)+B_{1} u(t)+B_{2} \delta(t)+B_{3} z(t)+\bar{B}_{1} \bar{u}(t)+B_{6} \phi(t) \\
y(t) & =C x(t)+D_{1} u(t)+D_{2} \delta(t)+D_{3} z(t)+\bar{D}_{1} \bar{u}(t)+D_{6} \phi(t)
\end{aligned}
$$

$$
E_{2} \delta(t)+E_{3} z(t) \leq E_{1} u(t)+E_{4} x(t)+E_{5}+\bar{E}_{1} \bar{u}(t)+E_{6} \phi(t)
$$

Here we explicitly split up the inputs used in nominal operation and the inputs for reconfiguration purposes as $u \in V_{N}$ and $\bar{u} \in V_{R}$, respectively. In nominal operation, we have $\phi=0$ and $\bar{u}=0$. Running the MPC optimization we obtain at each time step $t$ a triple $\left(u_{n}(t), \delta_{n}(t), z_{n}(t)\right)$. The state evolution in nominal operation is then given by

$$
x(t+1)=A x(t)+s_{n}(t)
$$

where $s_{n}(t)$ is determined online by the MPC controller and

$$
s_{n}(t)=B_{1} u_{n}(t)+B_{2} \delta_{n}(t)+B_{3} z_{n}(t)
$$

In the presence of fault $\phi_{i}$, we allow the use of the redundant inputs $V_{R}$. The state update is then given by:

$$
x(t+1)=A x(t)+s_{e}(t)
$$

where

$$
s_{e}(t)=B_{1} u_{e}(t)+B_{2} \delta_{e}(t)+B_{3} z_{e}(t)+B_{6} \phi(t)+\bar{B}_{1} \bar{u}_{e}(t)
$$

The key idea of this method is to make $s_{e}(t)$ as close to $s_{n}(t)$ as possible by exploiting the freedom we have to choose the redundant inputs $\bar{u}(t)$. This specification can be translated into the following optimization problem:

$$
\begin{array}{r}
J_{\mathcal{S}}=\min _{u_{e}, \bar{u}_{e}, \delta_{e}, z_{e}}\left\|s_{n}(t)-s_{e}(t)\right\|^{2} \\
\text { s.t. } E_{2} \delta(t)+E_{3} z(t) \leq E_{1} u(t)+E_{4} x(t)+E_{5}+\bar{E}_{1} \bar{u}(t)+E_{6} \phi(t)
\end{array}
$$

The algorithm is summarized next. Table 1 summarizes all the algorithms.

\section{Algorithm 5}

1. Run the MPC controller and the fault detection scheme in parallel. Compute the MPC control moves with the faultless model, yielding $u_{n}(t), \delta_{n}(t), z_{n}(t)$ and determine $s_{n}(t)$ from equation (16).

2. If a fault $\phi_{i}$ is detected, run the optimization (19), (20) to obtain $u_{e}(t), \bar{u}_{e}(t), \delta_{e}(t), z_{e}(t)$. If no fault is detected, set $u_{e}(t)=u_{n}(t), \bar{u}_{e}(t)=0, \delta_{e}(t)=$ $\delta_{n}(t), z_{e}(t)=z_{n}(t)$.

3. Apply $u_{e}(t)$ and $\bar{u}_{e}(t)$ to the system

4. Repeat 1. at the next time step.

\section{Reconfiguration of the Tank System}

We applied the controller reconfiguration ideas to the three tank benchmark system described in Section 2 . 
Table 1: Summary of the algorithms

\begin{tabular}{|l|l|l|}
\hline $\begin{array}{l}\text { Algo- } \\
\text { rithm }\end{array}$ & $\begin{array}{l}\text { Choice of candidate } \\
\text { inputs } V_{i}\end{array}$ & $\begin{array}{l}\text { Value of candidate } \\
\text { inputs } V_{i} \text { given by }\end{array}$ \\
\hline 1 & $V_{i}=V_{N} \cup V_{R}$ & MPC over $V_{i}$ \\
\hline 2 & look up table & MPC over $V_{i}$ \\
3 & static steady state & MPC over $V_{i}$ \\
4 & polytopic steady state & MPC over $V_{i}$ \\
\hline 5 & $V_{i}=V_{N} \cup V_{R}$ & correction $(19)-(20)$ \\
\hline
\end{tabular}

Due to lack of space we present only an application of Scenario 2. We assume that at time $t=30$ valve $V_{1}$ is stuck closed. When operated with the PI-controller, the tank system reacts to the fault as shown in Figure 4. Figure 5 (left) shows the application of algorithm 5 . Tank 2 is starting to be used when the fault is detected. Figure 5 (right) shows the application of algorithm 4 . After the failure, the controller switches the manipulated valve from $V_{1}$ to $V_{13}$. In this case the control goal can be achieved without using tank 2 , but the behaviour of $h_{1}$ is slightly worse than before. See [18] for more simulations and discussions.

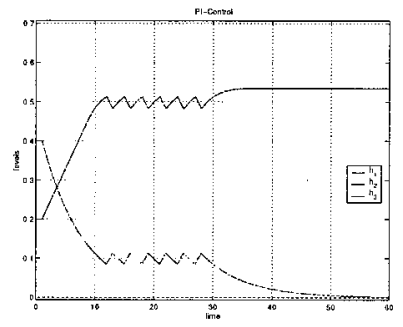

Figure 4: Scenario 2, nominal operation
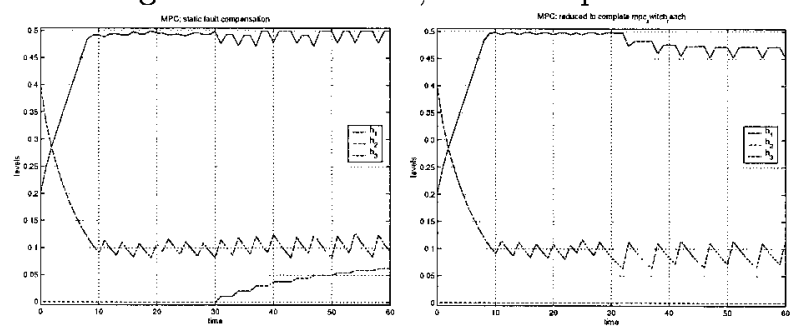

Figure 5: Scenario 2, static fault compensation (left) and polytopic steady state reconfiguration

\section{Conclusions}

In this paper we have dealt with the problem of choosing redundant manipulated variables for a system, which is subject to faults. This decision problem can be be formulated within the framework of mixed logic dynamical models and can be solved with mathematical optimization tools. The methods presented in this work aim at reducing the complexity of the reconfiguration problem, in order to render the problem tractable in an online supervision scheme.
Acknowledgement: This research is partially supported by the "Schweizer Bundesamt für Bildung und Wissenschaft" through "Esprit Project Verification of Hybrid Systems"

\section{References}

[1] J. Askari, B. Heiming, and J. Lunze. Controller Reconfiguration Based on a Qualitative Model: A Solution of ThreeTanks Benchmark Problem. Proceedings of the European Control Conference, Karlsruhe, Germany, 1999. Session CM5-3.

[2] A. Bemporad, F. Borrelli, G. Ferrari-Trecate, D. Mignone, M. Morari, and F.D. Torrisi. Classes and Properties of Mixed Logic Dynamical Systems. Technical report, Automatic Control Laboratory, ETH Zürich, Switzerland, 2000.

[3] A. Bemporad, G. Ferrari-Trecate, and M. Morari. Observability and Controllability of Piecewise Affine and Hybrid Systems. Proc. of the Conf. on Decision and Control, 1999.

[4] A. Bemporad, D. Mignone, and M. Morari. Moving Horizon Estimation for Hybrid Systems and Fault Detection. In Proceedings of the American Control Conference, 1999.

[5] A. Bemporad and M. Morari. Control of Systems Integrating Logic, Dynamics, and Constraints. Automatica, 35(3):407427, March 1999.

[6] J. Chen and R. Patton. Robust Model-Based Fault Diagnosis for Dynamic Systems. Kluwer Academic, 1998.

7] G. Ferrari Trecate, D. Mignone, and M. Morari. Moving Horizon Estimation for Piecewise Affine Systems. Proceedings of the American Control Conference, 2000.

[8] J. Gertler. Fault Detection and Diagnosis in Engineering Systems. Marcel Dekker, Inc., 1998.

[9] B. Heiming and J. Lunze. Definition of the Three-Tank Benchmark Problem for Controller Reconfiguration. Proceedings of the European Control Conference, Karlsruhe, Germany, 1999.

[10] E.C. Kerrigan, A. Bemporad, D. Mignone, M. Morari and J. M. Maciejowski. Multi-objective Prioritisation and Reconfiguration for the Control of Constrained Hybrid Systems. Proceedings of the American Control Conference, 2000.

[11] J. Lunze. Laboratory Three Tanks System - Benchmark for the Reconfiguration Problem. Technical report, Tech. Univ. of Hamburg-Harburg, Inst. of Control. Eng., Germany, 1998. http://www tu-harburg.de/rts/software/cosy/.

[12] J. Lunze and J. Schröder. Qualitative Diagnosis of the 3Tanks System. Proceedings of the European Control Conference, Karlsruhe, Germany, 1999.

[13] T. Marcu, M. H. Matcovschi, and P.M. Frank. Neural Observer-Based Approach to Fault-Tolerant Control of a ThreeTank System. Proceedings of the European Control Conference, Karlsruhe, Germany, 1999. Session CM5-5.

[14] D. Mignone. Moving Horizon Estimation and Fault Detection of Mixed Logic Dynamical Systems. Postdiploma Thesis (Nachdiplomstudium Informationstechnik), Automatic Control Laboratory, ETH Zürich, Switzerland, 1999.

[15] G.L. Nemhauser and L.A. Wolsey. Integer and Combinatorial Optimization. Wiley, 1988.

[16] R. Patton. Robustness Issues in Fault-Tolerant Control. In Colloquium on: "Fault Diagnosis and Control System Reconfiguration"; organised by Professional Group C8, 1993. Savoy Place, London, UK.

[17] L. Rato and J.M. Lemos. Multimodel Based Fault Tolerant Control of the 3-Tank System. Proceedings of the European Control Conference, Karlsruhe, Germany, 1999. session CM5-4. [18] K. Tsuda, D. Mignone, G. Ferrari-Trecate, and M. Morari. Reconfiguration for mixed logic dynamical systems. Technical report, Automatic Control Laboratory, ETH Zürich, Number AUT00-24, 2000. 